\title{
Peuplement Spontané Et Accès À L'éducation Primaire Dans La Commune De Bantè Au Centre Du Bénin
}

\begin{abstract}
Akobi K.
Innocent, Département de Géographie et Aménagement du Territoire de l’Université d’Abomey Calavi (UAC), Laboratoire Pierre PAGNEY

'’Climat, Eau, Ecosystème et Développement (LACEDE), Benin

\section{Yabi Ibouraima}

Département de Géographie et Aménagement du Territoire de l’Université d’Abomey Calavi (UAC), Laboratoire Pierre PAGNEY '’Climat, Eau, Ecosystème et Développement (LACEDE), Benin
\end{abstract}

\section{Agnon Nacisse}

Département de Géographie et Aménagement du Territoire de l’Université de Parakou (UP), Bantè, Benin

\section{Amoussou Ernest}

Département de Géographie et Aménagement du Territoire de l’Université d’Abomey Calavi (UAC), Laboratoire Pierre PAGNEY'’ Climat, Eau,

Ecosystème et Développement (LACEDE), Benin

\section{Boko Michel}

Département de Géographie et Aménagement du Territoire de l’Université d’Abomey Calavi (UAC), Laboratoire Pierre PAGNEY, Laboratoire Pierre PAGNEY '’Climat, Eau, Ecosystème et Développement (LACEDE), Benin

doi: 10.19044/esj.2017.v13n19p168 URL:http://dx.doi.org/10.19044/esj.2017.v13n19p168

\section{Abstract}

Achieving universal primary education is the second Millennium Development Goals that Benin missed in 2015 with a national rate of 74 percent. The reasons for this failure are varied with notable spatial specificities. The present research aims to contribute to the analysis of the specific causes of this failure in the Bantè Commune. The data used relate to the number of children enrolled in schools, the number of schools, and the number of settlements (villages, hamlets, and manned farms) on several dates in the Commune. In addition, information was collected from selected households in several localities. Individual interviews and groups as well as direct field observations were the collection techniques used. The tools and parameters of the descriptive statistics and the 
cartographic approach made it possible to process the data collected. The results show a disparity in the spatial distribution of primary schools compared to inhabited places. The result shows a geographical inaccessibility of schools for the children of many hamlets and farms. This situation is mainly inherent to a spontaneous settlement dynamics which characterizes the Commune. This concern, therefore, deserves consideration if access to primary education is a global challenge that is again relaunched by 2030. This is usually done within the framework of the Sustainable Development Goals.

Keywords: Benin, Bante, primary education, spontaneous settlement, MDG, ODD

\section{Résumé}

Assurer l'éducation primaire pour tous, est le deuxième des Objectifs du Millénaire pour le Développement que le Bénin a raté en 2015 avec un taux national de 74\%. Les raisons qui expliquent cet échec sont diverses et variées avec des spécificités spatiales notoires. La présente recherche vise à contribuer à l'analyse des causes spécifiques de cet échec dans la Commune de Bantè. Les données utilisées se rapportent à l'effectif des enfants scolarisés, le nombre d'établissements scolaires, le nombre d'établissements humains (villages, hameaux et fermes habités) à diverses périodes dans la Commune. A cela s’ajoutent des informations collectées auprès des ménages. Des entretiens individuels et de groupes de même que des observations directes de terrain ont été les techniques de collecte utilisées. Les outils et paramètres de la statistique descriptive et l'approche cartographique ont permis de traiter les données collectées. Les résultats montrent une disparité dans la répartition spatiale des écoles primaires par rapport aux lieux habités. Il en résulte donc une inaccessibilité géographique des écoles pour les enfants de nombreux hameaux et fermes. Cette situation est principalement inhérente à une dynamique de peuplement spontané qui caractérise la Commune. Cette préoccupation mérite donc réflexion si tant est que l'accès à l'éducation primaire est un défi planétaire qui est à nouveau relancé pour l’horizon 2030 dans le cadre des Objectifs de Développement Durable.

Mots-clés: Bénin, Bantè, éducation primaire, peuplement spontané, enfant scolarisé

\section{Introduction}

Les travaux de Droy (2004) ont montré que la scolarisation a des conséquences directes sur le niveau de formation acquis et donc sur le capital 
humain, éducation que peuvent valoriser par la suite les individus. Ainsi donc, depuis la détermination de l’objectif Education Pour Tous (EPT), défini à Jomtien en 1990, lequel objectif est réaffirmé et décliné en stratégies en 2000 lors du forum de Dakar, l’option d'un développement accéléré de l'éducation de base est prise par la communauté internationale (Bourdon, 2006). Selon le même auteur, cette stratégie reconnaît une priorité d'affectation de l'aide internationale pour financer l'éducation, là où la pauvreté des pays les empêche de rattraper l’écart avec les pays développés. C'est dans cette logique que l'Organisation des Nations-Unies a consacré l'éducation humaine au rang des priorités dans le cadre des Objectifs du Millénaire pour le Développement (OMD) où elle a fixé comme $2^{\text {ème }}$ objectif d'assurer l'éducation primaire pour tous en 2015.

Mais en Afrique, les efforts d'amélioration des niveaux de vie et de la croissance économique et le développement de la capacité technologique butent sur plusieurs difficultés qui sont d'ordre structurel et conjoncturel (Touré, 2010). Au nombre de ces difficultés figure en bonne place la non maîtrise de l'occupation spatiale qui ne facilite pas l'accessibilité de tous aux infrastructures et de ce fait affecte négativement les efforts d'investissements. En effet, depuis l'apparition de l'Homme sur la Terre, le peuplement humain a toujours revêtu un caractère dynamique (Penchmel, 1992). La non maîtrise de ce fait de société entrave considérablement les efforts de développement des nations (Baudelle, 2000). Les pays en voie de développement éprouvent d’énormes difficultés parmi lesquelles figurent en bonne place celles relatives à l’éducation primaire (ONU Bénin, 2016).

Le Bénin n’échappe pas à cette tendance peu reluisante, en dépit des efforts qui sont déployés ces deux dernières décennies. Ainsi, l'évaluation des progrès accomplis par le Bénin en matière d'éducation, dans la réalisation des Objectifs du Millénaire pour le Développement (OMD) entre 2000 et 2015, a relevé des insuffisances et des disparités spatiales en matière d'accès à l'éducation primaire notamment.

La présente recherche vise à appréhender les facteurs explicatifs de l'accès limité à l'éducation primaire dans la Commune de Bantè et se fonde sur la question principale suivante: quelles sont les implications du peuplement spontané (non planifié) sur l'accès des enfants en âge de scolarisation dans la Commune de Bantè? L'hypothèse associée à cette réflexion est que le peuplement non planifié dans un contexte de moyens limités ne favorise pas l'accès de tous les enfants aux infrastructures scolaires disponibles. Cette contribution est à cheval entre la géographie de la population et l'aménagement du territoire dans un contexte où l'accès des communautés aux services sociaux de base préoccupe les décideurs dans les pays en voie de développement comme le Bénin. 


\section{Approche Méthodologique et Cadre Géographique D’étude Méthodes et Matériels de Travail Méthodes}

La méthodologie utilisée repose sur la recherche documentaire d'une part, les travaux de terrain, le traitement des données et l'analyse des résultats, d'autre part. Le croisement des analyses chronologique et diachronique de l'histoire du peuplement de cet espace géographique a amené à un découpage temporel de son processus.

\section{- $\quad$ Recherche documentaire}

La recherche documentaire a permis de faire la synthèse des documents disponibles et d'analyser quelques ouvrages spécifiques relatifs au sujet, objet de la recherche. Ainsi, les documents ont été consultés dans les centres de documentation de la Faculté des Lettres, Arts et Sciences Humaines (FLASH), de la Faculté des Sciences Agronomiques (FSA), de l'Institut National de la Statistique et de l'Analyse Economique (INSAE), l'Institut Géographique National (IGN), du Ministère en charge de la Décentralisation et l'Aménagement du Territoire, de la Mairie de Bantè, de la Circonscription Scolaire sans oublier l'Internet. Les informations documentaires ainsi mobilisées ont été complétées par les travaux de terrain.

\section{- $\quad$ Travaux de terrain}

Ils regroupent les observations, les enquêtes en milieu réel, la prise de différentes mesures et de photos. Les enquêtes ont été orientées vers les groupes cibles avec un échantillonnage par groupe socioprofessionnel dans une proportion variable. Il s’agit des élus communaux, locaux et agents de la mairie (10\%), des enseignants de la maternelle et du primaire, aussi bien du public que du privé (25\%), des parents d'élèves (40\%), des membres des Associations des Parents d'Elèves (15\%), des écoliers (8\%) et enfants déscolarisés (2\%). Au total 350 personnes ont été interrogées par questionnaire. L’entretien par focus group a été aussi réalisé auprès des populations allochtones de 30 hameaux et fermes sur 190 recensés, soit 15\% du total.

La prise de mesures concerne les coordonnées géo-référencées utiles pour l'élaboration des cartes.

\section{- Travaux cartographiques}

Ils ont consisté à la réalisation des cartes de peuplement mettant en relief le semi continu des lieux habités dans le temps et dans l'espace. Les cartes d'équipements scolaires et d'accessibilité géographique ont été réalisées sur la base des données collectées au bureau du chef de la circonscription scolaire à Bantè. Les données démographiques utilisées sont celles de l'INSAE des années1979, 1992, 2002 et 2013. 
- $\quad$ Traitement des données et analyse des résultats

Pour mieux appréhender les problèmes que soulève ce sujet et en faire une bonne interprétation, la technique d'analyse basée sur l'outil SEPO (Succès, Echec, Potentialité, Opportunité) a été adoptée. Les matrices Succès et Opportunité ont permis d'analyser les efforts du gouvernement et de la Commune pour l'atteinte des OMD tandis que celles des Echec et Potentialité ont favorisé le diagnostic des handicaps de ce challenge.

Ces travaux de terrain et analyses des résultats ont été menés à bien, grâce à l'utilisation de certains matériels.

\section{Matériels de Travail}

Les enquêtes de terrain ont été réalisées à l'aide de plusieurs outils, notamment : différentes cartes (topographique, occupation du sol, etc.); un questionnaire à l'endroit des populations; un guide d'entretien pour collecter des informations auprès des personnes ressources; une fiche de relevé des coordonnées géographiques; le logiciel SPSS; un appareil photographique numérique pour la prise des vues sur le terrain; un GPS (Global Positioning System) pour la prise des coordonnées géographiques des localités et équipements scolaires; etc.

Avant de présenter les résultats de cette recherche, il importe de mettre brièvement en relief le cadre d'étude.

\section{Cadre Géographique de L'étude}

Située entre $8^{\circ} 4^{\prime}$ et $8^{\circ} 37^{\prime}$ de latitude Nord et $1^{\circ} 36^{\prime}$ et $2^{\circ} 11^{\prime}$ de longitude Est (figure 1), la Commune de Bantè est située au nord-ouest du Département des Collines et est limitée au Nord par la Commune de Bassila (Département de la Donga), au Sud par la Commune de Savalou, à l'Est par la Commune de Glazoué et à l'Ouest par la République du Togo. Elle couvre une superficie de $2695 \mathrm{~km}^{2}$ et abrite une population de 107181 habitants (RGPH 4, 2013). 


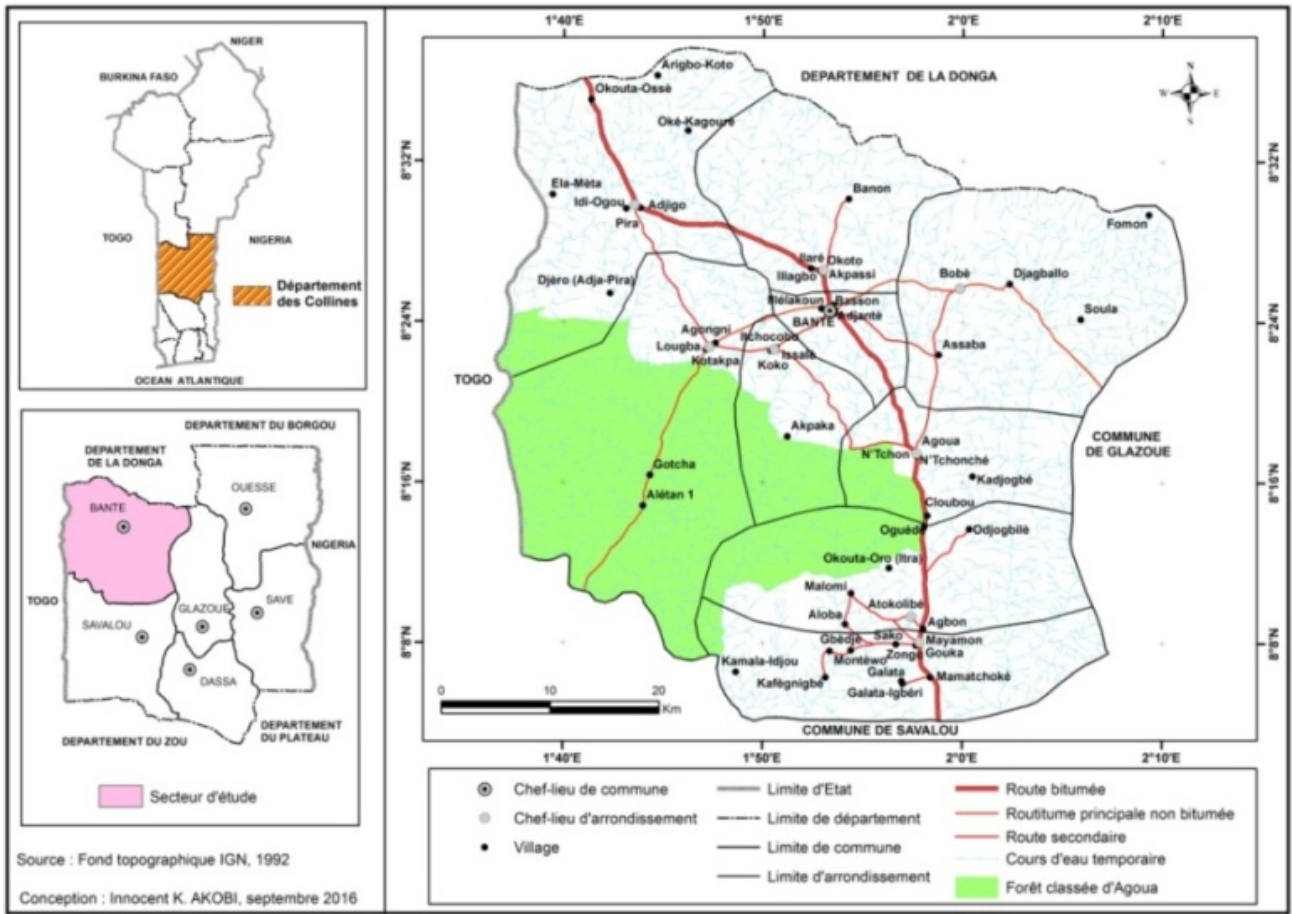

Figure 1. Situation géographique de la Commune de Bantè

La méthodologie utilisée dans le cadre de cette étude nous a permis d’obtenir des résultats qu’il importe de présenter.

\section{Résultats et Discussions}

Ils portent sur les facteurs favorables à la mise en place des populations de la Commune de Bantè, l'historique du peuplement, la politique du gouvernement en matière de la promotion de l'éducation à la base et l'impact de la dynamique de peuplement spontané sur l'éducation primaire dans cette Commune.

\section{Facteurs Favorables à la Dynamique de Peuplement}

Les facteurs qui ont favorisé ce type de peuplement sont divers et variés et sont aussi bien endogènes qu'exogènes. Il s'agit des facteurs physiques, des facteurs humains et ceux économiques, tous en interaction étroite.

\section{Facteurs Physiques Favorables}

Les éléments de la nature qui ont favorisé la dynamique actuelle du peuplement de la Commune de Bantè sont liés à la pluviosité, la pédologie, l'hydrographie et la végétation du milieu qui sont relativement propices au développement des activités agricoles et pastorales. 
L'évolution des hauteurs de pluie de la Commune de 1951 à 2010 a permis de déterminer les années excédentaires (humides), déficitaires (sèches), et normales (stables). Ainsi, la moyenne arithmétique inférieure est de 926,76 mm tandis que celle supérieure de $1390 \mathrm{~mm}$. La figure2 révèle que sur la période 1951-2010, seules les années 1967et 2007 sont les plus excédentaires et les années 1958 et 1990 sont les plus déficitaires. A ce contexte pluviométrique dont est tributaire un couvert végétal assez luxuriant en saison pluvieuse, il faut associer les sols largement ferrugineux et partiellement ferralitiques et hydromorphes qui sont dans leur ensemble appropriés aux cultures vivrières (maïs, haricot, mil), aux tubercules (igname, manioc) et aux cultures de rente (coton, anacarde).

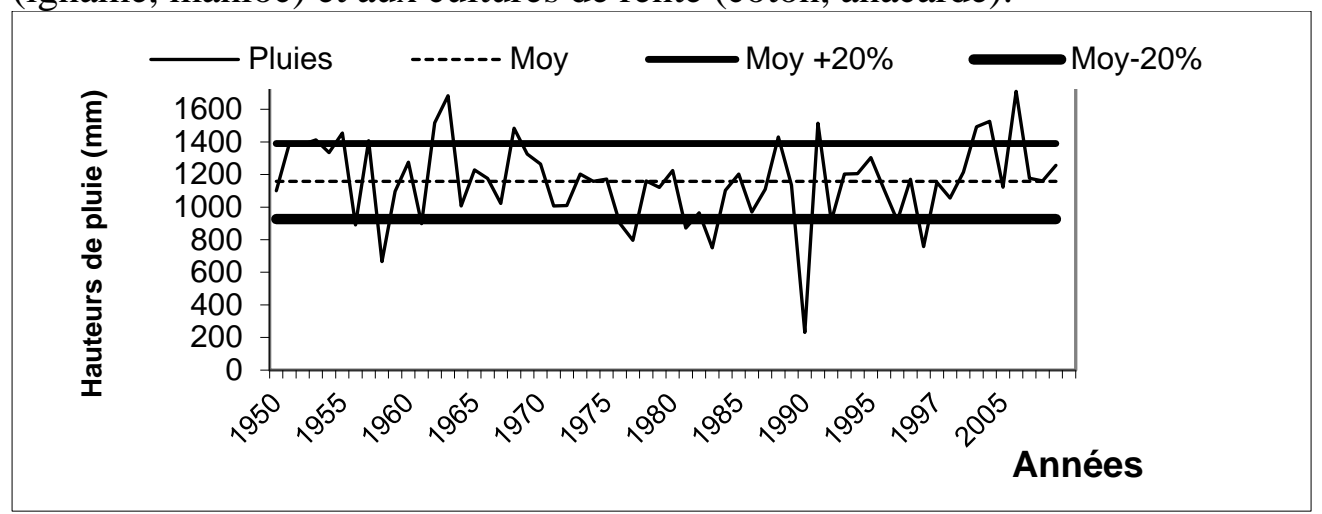

Figure 2. Variation interannuelle des hauteurs pluviométriques (1951-2010)

Source: ASECNA, 2016

Par ailleurs, il faut souligner que les sécheresses des années 1970 et 1980 qui ont sévit dans la partie septentrionale ont obligé les populations agricoles de ces milieux à migrer vers la Commune de Bantè où les conditions climatiques paraissent moins austères.

\section{Facteurs Humains et Socioculturels Propices}

Ils touchent la faible densité de population de la Commune qui serait due en partie à un certain nombre de faits historiques, notamment les razzias esclavagistes et les guerres tribales. C'est le vide laissé par les autochtones qui se comble aujourd'hui par les immigrants venus d'ailleurs. Par contre, c'est la forte densité de population de la partie sud du Bénin (entre 100 et 1000 habitants au $\mathrm{km}^{2}$ ) avec pour corollaire la réduction de la taille des exploitations agricoles des ménages qui justifie l'installation des colons agricoles venus de cette région. Ces flux migratoires ont sans doute renforcé la croissance démographique de la Commune dont l'évolution se présente comme l'indique la Figure 3. 


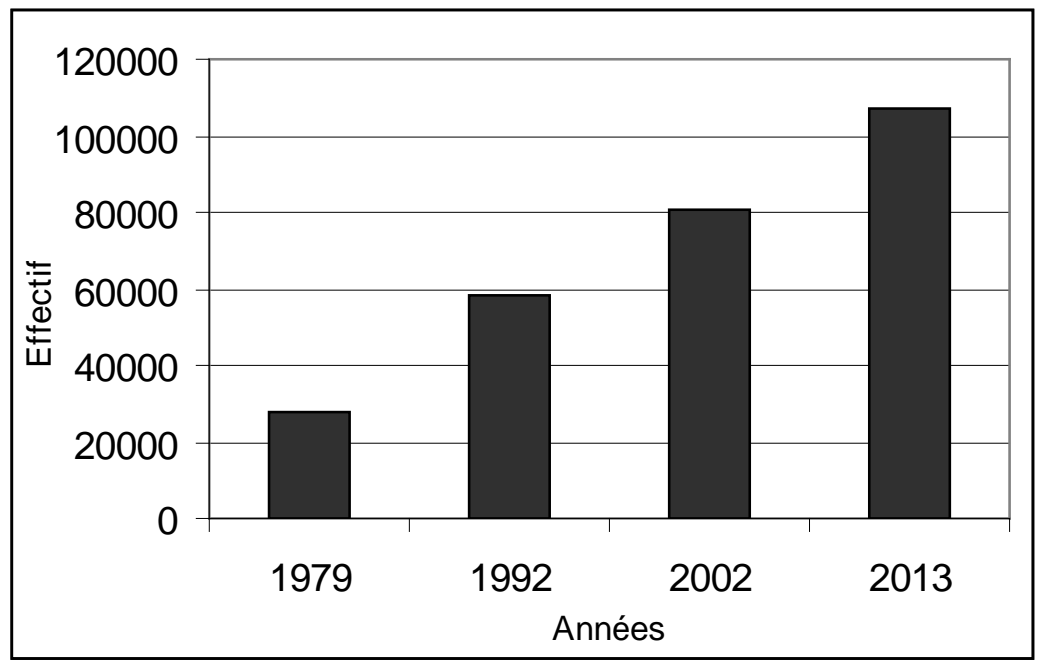

Figure 3. Evolution de la population de la commune de Bantè Source: Réalisé à partir des données des RGPH 1, 2, 3, et 4

La Figure 3 montre qu'en 34 ans, la population de la Commune a presque quadruplé passant de 28000 en 1979 à 107181 habitants en 2013. Or, plus de $75 \%$ de la population pratiquent des activités agricoles ou agropastorales et exercent donc une forte pression sur les ressources foncières. Aussi, l'hospitalité légendaire des autochtones est-elle favorable à la cohabitation et l'intégration des allochtones. En effet, dans la culture des Isha et Ifè, la terre est sacrée donc inaliénable ne devrait pas faire l'objet de vente. C'est pourquoi, pendant longtemps, elle est cédée gratuitement aux allochtones qui en sollicitent pour cultiver.

Par ailleurs, les pratiques culturales faites de l'itinérance sur brûlis à l'aide des feux de végétation reposent sur une agriculture extensive consommatrice de l'espace (Akobi, 2002; Oloukoï, 2012, \& Arouna, 2012). Ces pratiques occasionnent l'occupation lâche de la terre et induit la dispersion des lieux habités (Baudelle, 2000). En outre, l'effritement des valeurs socioculturelles caractérisé par la déstructuration du tissu social des communautés face à la modernité a engendré l'individualisme (Thomas, 2014). Les grandes exploitations agricoles des collectivités ont progressivement cédé place aux domaines des familles nucléaires. D’où la prolifération des fermes créées par des ménages autochtones à l'instar des allochtones.

\section{Considérations économiques}

Les facteurs économiques qui favorisent la dynamique de peuplement de la Commune de Bantè ont trait à l'essor économique de deux principales cultures de rente au début des années 1990. En effet, les mesures de 
redressement économique prises sous l'ère du renouveau démocratique, ont relancé la filière coton et la filière anacarde par effet d'entrainement (association des deux cultures). Il en a résulté l'extension des domaines exploités et l'amélioration substantielle des revenus des paysans. De ce fait, les paysans sont obligés de faire appel à la main d'œuvre agricole extérieure. Cette période de prospérité retrouvée a stabilisé les autochtones candidats à l'exode rural et a contribué à un développement spectaculaire des plantations d'anacarde que les paysans qualifient de 'pension de retraite'. C'est ce qui vaut à la commune de Bantè la première place dans la production de l'anacarde au niveau national avec une production annuelle oscillant entre 25000 et 30000 tonnes (SCDA Bantè, 2014).

La combinaison de ces facteurs détermine les caractéristiques de la dynamique du peuplement de ce territoire.

\section{Peuplement du territoire de la Commune de Bantè: Un Processus Long, Dynamique, et Spontané}

Les enquêtes de terrain complétées par les recherches documentaires ont permis de découper le processus de peuplement du territoire de l'actuelle Commune de Bantè en trois grandes périodes de durée et d'intensité inégales à savoir: la période précoloniale, la période coloniale et la période postcoloniale (Figure 4).

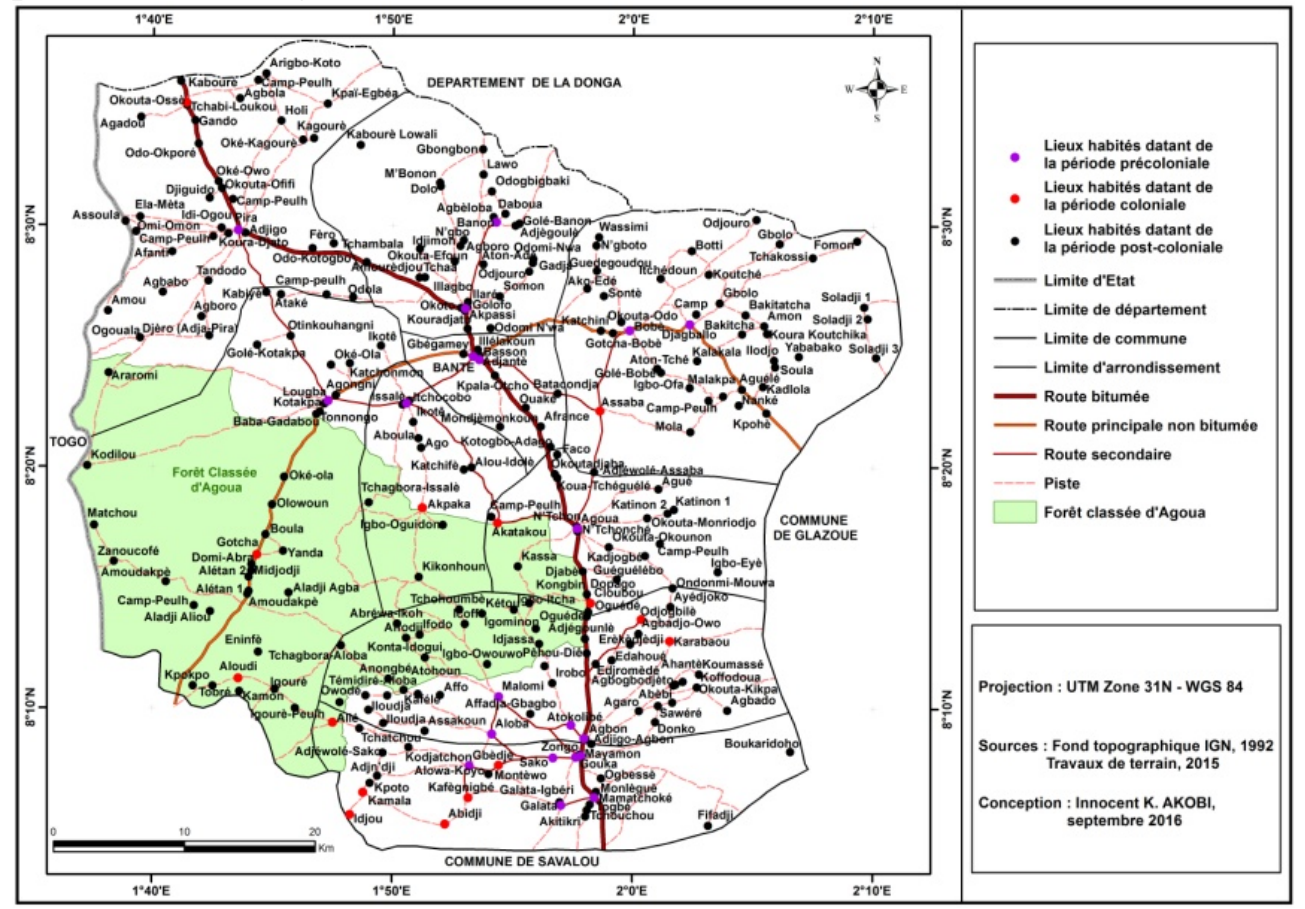

Figure 4. Carte de la dynamique de peuplement de la Commune de Bantè Sources : Recherches documentaires et enquêtes de terrain (2013-2016) 


\section{Peuplement Pendant la Période Précoloniale}

La période précoloniale, la plus longue, est celle de l'installation des peuples Isha et Ifè venus respectivement de '’Ilé-Sha' et '’llé-Ifè' de l'actuel Nigéria, vers le XII ${ }^{\mathrm{e}}$ siècle (Adam \& Boko, 1983). C’est une période très mouvementée puisqu'elle est marquée par les guerres tribales et les razzias esclavagistes. Il en a résulté, un transfert massif des populations de cette partie du Bénin vers la côte atlantique et l'Amérique (Cornevin, 1965). L’insécurité caractéristique de cette période a favorisé le peuplement à habitat groupé des autochtones. Les premiers lieux habités sont Adjantè, Lougba, Koko, Lougba, Banon, Akpassi, Djagbalo, Bobè, pour les Isha au nord et les localités d’Ilodji que sont Gouka, Mamatchoké, Galata, Mayamon, Agbon, Atokolibé, Malomi, Aloba et Sako pour les Ifè au sud (Figure 4).

\section{Peuplement Au Cours la Période Coloniale}

La période coloniale quant à elle marquée par la pénétration et la domination de la puissance coloniale est caractérisée par une stabilité sociopolitique relative. Cette période pacifique a favorisé l'éclosion des premiers lieux habités pour donner naissance aux lieux habités de la période coloniale (Figure 4). Il s’agit des localités de Assaba, Akatakou, Gotcha, Alétan, Okouta-Ossé, Kagourè, Okouta-Adjaba pour le peuple Isha au nord et les localités de Gbèdjè, Kafégnigbé, Montèwo, Idjou, Alé, Kamala, Abidji pour les Ifè au sud. Au cours de cette période, on note la pénétration de l'aire culturelle Isha-Ifè par les premiers immigrants dahoméens. Ce qui a donné naissance à des localités de Odjogbilè, Krabahou, Clougbou et Kadjogbé. Par ailleurs, les peuples Isha-Ifè ont connu durant cette période à l'instar des peuples voisins Mahi, Idaasha et Sabè, le départ des bras valides en direction des pays de la sous-région notamment, le Ghana et la Côte-d’Ivoire à la recherche du bien-être (Akobi, 1997).

\section{Peuplement Pendant la Période Postcoloniale}

La troisième période, la moins longue, est celle postcoloniale qui est caractérisée par d’importants flux migratoires. On note au cours de cette période l'arrivée de nombreux immigrants du sud et du nord Bénin d'une part, le retour dans les années 1960 des émigrés rapatriés du Ghana et de la Côte-d'Ivoire et aussi le départ massif des autochtones en direction du Nigéria au début des années 1980 d’autre part. Cette période postcoloniale peut se subdiviser en deux phases. La première phase, est celle qui va de 1960 à 1990 et la deuxième, de 1990 à nos jours.

En effet, cette dernière période est celle de l'éclosion de nombreuses fermes créées aussi bien par les autochtones que par les allochtones, surtout à partir des années 1990. En fait, depuis quelques décennies, la Commune de 
Bantè, à l'instar des autres Communes du Moyen-Bénin, reçoit d'importantes vagues de migrants des groupes socioculturels venus du sud (Adja, Fon, Holli...), du centre (Idaasha, Mahi...), du nord Bénin (Lokpa, Otamari, Pila-pila...) et même des pays voisins (Kotokoli, Kabyè, Peulh). Ces migrants qui s’adonnent pour la plupart aux activités agropastorales créent des fermes et campements ou cohabitent avec les autochtones. On peut citer des fermes et hameaux tels que Gando, Elamèta, Adja-Pira, Otinkouagni, Fomon, Soula, Aguélé, Fèro-Iya, Ouaké, Edahoué, Péhoudiè, Afrance, Akando, etc. (figure 4). Par ailleurs, depuis quelques années, ce sont les Ifè du sud de Bantè qui sont contraints par la rareté de terres cultivables à migrer vers le nord-est de la Commune de Bantè, précisément dans la zone de Djagbalo pour créer de nouvelles fermes de cultures (Akobi, 2002).

De ce qui précède, il faut dire que la mise en place des peuples du territoire de la Commune de Bantè s’est faite progressivement dans le temps et dans l'espace. A sa création en 1978 comme District rural, on y dénombrait trente-quatre (34) villages administratifs dont quatre (4) quartiers de ville. Avec l'actualisation de la carte administrative réalisée en 2013, la Commune compte désormais quarante-neuf (49) villages dont quatre (4) quartiers de ville. La plupart des lieux habités créés au cours de la période coloniale sont érigés en unités administratives. Mais à toutes ces unités administratives se rattachent aujourd'hui près de deux cents (200) fermes et hameaux. Ce peuplement qui n’épargne même pas le domaine classé de l'Etat (Figure 4), se caractérise par la spontanéité de l’occupation spatiale. D’après les enquêtes, aucune mesure d'organisation de la colonisation agricole n’est prise par les gouvernants, contrairement à ce qui se passe dans certains pays africains où l'Etat et les organismes humanitaires accompagnent ce phénomène (Moupou, 2010; Notope, 2010). Plus de 96\% des enquêtés ont témoigné de l'irrationalité de ce peuplement. Les résultats de recherche de Sounon (2011) et de Oloukoï (2012) ont également abouti à cette conclusion.

$\mathrm{Au}$ total, les facteurs physiques, humains et économiques non seulement maintiennent les autochtones, mais aussi attirent d'autres groupes socioculturels qui affluent. Il en résulte une dynamique de peuplement qui a abouti à la prolifération des lieux habités dont l'importance numérique, l'éloignement, l'enclavement impactent négativement l'éducation primaire en dépit des mesures salvatrices prises par le gouvernement béninois dans la période 2000-2015 dans le cadre des OMD. 


\section{Mesures Institutionnelles en Faveur de L’éducation au Bénin : Portée et Limites \\ Mesures Volontaristes Prises}

$\mathrm{Au}$ nombre de ces mesures prises au niveau national, quatre principales sont mises en relief.

- L'accès à l'école et le maintien dans le système, notamment chez les filles

L'accès à l'école et le maintien dans le système sont portés, depuis octobre 2006, par les mesures de gratuité à l'école maternelle et primaire (non-paiement des frais d'inscription). Ces mesures sont accompagnées au niveau national du transfert de ressources aux Communes pour permettre la construction et l'équipement de salle de classes chaque année, de l'instauration de la subvention au profit des écoles primaires publiques pour couvrir leurs frais de fonctionnement, de recrutement de 9854 enseignants entre 2006 et 2014, du reversement dans la fonction publique de tous les enseignants communautaires et contractuels locaux comme Agents Contractuels de l'Etat, de vastes campagnes de sensibilisation organisées dans les Communes à faible taux de scolarisation des filles, de l'appui en fournitures scolaires aux enfants issus des couches sociales défavorisées ou des familles pauvres.

- La promotion d'initiatives écoles privées dans le domaine de l'éducation

Elle a permis d'accroître le pourcentage d'élèves dans le privé ces dernières années de $11,8 \%$ en 2005 à $13,2 \%$ en 2010 , jusqu'à $18,5 \%$ en 2014. Ces initiatives privées ont été également prises au niveau de la commune de Bantè, comme on le verra plus loin. Le développement du secteur privé dans l'éducation contribue à l'amélioration de l'offre éducative à travers l'augmentation des capacités d'accueil et donc, du niveau de scolarisation sur le plan national. Mais il faut signaler que l'éducation alternative mise en œuvre par le Plan stratégique de Programme de Cours Accéléré en vue de donner une deuxième chance aux enfants déscolarisés ou non scolarisés, n’a pas connu de succès dans la Commune de Bantè.

- Le maintien des élèves jusqu'à la fin du cycle primaire favorisé par des mesures sociales d'envergure

Le développement quantitatif du système éducatif béninois s'est accompagné d'importantes déperditions avec un fort taux d'abandon (de 2010 à 2014, le taux d'abandon est passé de 10,72\% en 2010 à 11,65\% en 2014), taux qui s'accroît au fur et à mesure que le niveau d'enseignement augmente. L'amélioration de l'accès jusqu'à la fin du cycle primaire se fait, notamment avec :

-le développement de l'enseignement maternel avec l'appui des partenaires tels que le Programme Alimentaire Mondial (PAM), les ONG 
nationales et internationales et les communautés à la base, qui participent à l'éveil des enfants ;

- le programme d'alimentation scolaire qui permet d'attirer et de retenir les enfants à l'école et de les sortir du cycle de la faim avec notamment l'appui du PAM ;

-la mesure dite des sous-cycles qui interdit le redoublement à l'entrée des trois cycles que compte le cycle primaire au Bénin (CI, CE1 et CM1) ;

- la réduction de la discontinuité éducative qui est l'une des sources de la déperdition scolaire et l'enseignement multigrade qui a été expérimenté dans ce programme de discontinuité éducative pour gérer de façon efficiente, les problèmes des écoles à faible effectif dans les classes et d'insuffisance d'enseignants et de salles de classe.

- L'amélioration de la qualité des intrants portée par des mesures sociales et la formation des enseignants

Pour assurer une éducation primaire de qualité, plusieurs actions ont été exécutées au cours de la période 2000 à 2015, à savoir entre autres :

- la politique nationale de formation élaborée et validée par le gouvernement qui renferme la formation initiale des enseignants tant dans le secteur public que le privé, d'une part, leur formation continue et leur encadrement pédagogique, d'autre part ;

- la distribution des manuels aux écoliers qui a permis d'améliorer les ratios des manuels de français et de mathématique par élève.

\section{D’importantes Ressources Mobilisées Pour Accroître Les Performances}

Le niveau appréciable des indicateurs de l'OMD 2 est le fruit des efforts conjugués de l'Etat et des PTF. Ces efforts se traduisent par la mise à disposition d'importantes ressources. La part des dépenses de l'Etat allouée au secteur de l'éducation est passée de $16,5 \%$ à 24,8\% sur la période 2000 2010, soit 3,1\% à 5,4\% du PIB. Le programme Fast-Track, dont a bénéficié le Bénin dans la période 2008-2013 au profit de la maternelle et du primaire s'est élevé à plus de 20 milliards FCFA. Ces ressources additionnelles à celles de l'Etat ont permis d'accélérer la mise en œuvre du Plan Décennal de Développement du Secteur de l'Education (PDDSE). En outre, le Fonds Commun Budgétaire (FCB) mis en place avec la DANIDA, l’AFD, la KFW, les Pays-Bas et la Banque mondiale a apporté, sous forme d'appui budgétaire, un financement complémentaire au Fonds Catalytique de l'initiative FAST-TRACK. Cette contribution à l'Education Pour Tous (EPT) au Bénin d'ici à 2015 s'est élevée à 31,741 milliards de FCFA. De plus, les agences de coopération multilatérale, notamment des Nations-Unies (UNESCO, UNICEF, PAM, etc.) et des ONG internationales ont accompagné la mise en œuvre des plans stratégiques de développement du secteur par des appuis techniques et financiers. Toutefois, il importe de 
signaler que le secteur a connu un gap de financement d'au moins 38,387 milliards de FCFA qui a contribué à freiner l'atteinte de l'OMD2 en 2015.

\section{Dysfonctionnements Ayant Freiné L’atteinte de l’OMD2}

L'évaluation du PDDSE ainsi que l'audit institutionnel et organisationnel du secteur de l'éducation, l'actualisation du Rapport d'Etat du Système Educatif National, l'étude diagnostique de la question enseignante au Bénin et l'évaluation de la formation dans les écoles de formation des enseignants (ENI et ENS) ont mis en évidence de nombreux dysfonctionnements du système éducatif. Il s’agit de:

- le trop peu d’efforts consacrés à la qualité, comparés à ceux faits pour l'accès, la rétention, et l'équité dans le système éducatif;

- le manque de ciblage des poches de résistance en matière d’accès et de rétention;

- la perturbation du système éducatif par des crises scolaires devenues cycliques, des grèves récurrentes entravent l'apprentissage dans les écoles;

- la pénurie de personnels, qui agit sur la qualité de l’enseignement (le ratio élèves/maître qui était de 50 en 2005 est de 53,1 en 2014), mais aussi la répartition géographique de ceux-ci (moins nombreux dans les zones rurales);

- le manque de professionnalisme de certains enseignants;

- l’insuffisance et l'inaccessibilité des infrastructures;

- la forte pression démographique.

Au regard de ce qui précède, quelle peut être la situation de l'éducation primaire à Bantè ?

\section{Retombées Positives Dans la Commune de Bantè Mais Limitées Par la Dynamique d'un Peuplement Spontané}

L’impact de cette dynamique du peuplement sur l'éducation ne peut se mesurer qu'à travers les difficultés d'accès des enfants à l'école du fait de l’éloignement de leur lieu de résidence aux localités disposant d’école.

\section{Retombées Des Mesures Prises Par l'Etat Béninois sur L’éducation Primaire Dans la Commune de Bantè}

Les mesures prises par l'Etat ont affecté positivement la Commune sur plusieurs volets.

$\checkmark \quad$ Du boom d'écoliers, à la prolifération d'écoles et d'enseignants contractuels

L'ensemble des mesures prises par l'Etat central sus-évoquées ont positivement impacté l'éducation primaire dans la Commune de Bantè surtout dans les villages dans la période 2000-2015.Par rapport à l'effectif 
des écoliers dans le public, les chiffres sont passés de 14 183en 2000 à 25 219 apprenants en 2012 (Figure 5).

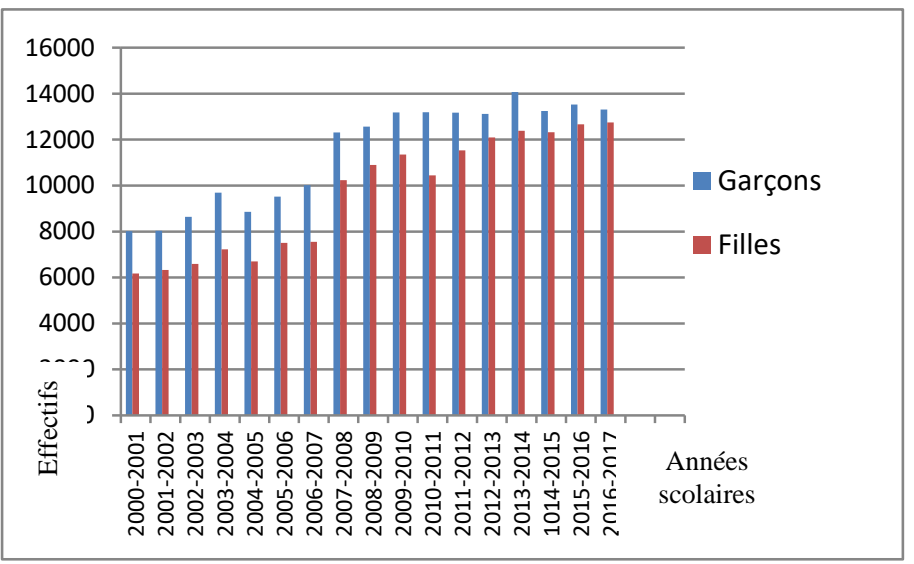

Figure 5. Evolution de l'effectif des écoliers par sexe dans la Commune de Bantè (20002017)

Source: Archives de la Circonscription Scolaire de Bantè

Dans le même temps on a enregistré beaucoup de création ou d'extension d'écoles primaires publiques et privées. En 2016, la Commune de Bantè compte au total 107 écoles primaires publiques, 27 écoles maternelles publiques, 13 écoles primaires privées et 2 écoles maternelles privées.

Sur un total de 107 écoles primaires publiques que compte la Commune de Bantè, 42 sont créées entre 2000 et 2015, soit près de $40 \%$ en 15 ans (la première étant créée en 1940).Pour ce qui est des écoles maternelles, 26 sur les 27 que compte la Commune sont créées dans cette période, soit $96 \%$.

Pour ce qui concerne les écoles primaires privées, sur 13 que compte la Commune en 2016, il ya 11 qui sont créées entre 2000 et 2015 ; les 2 autres qui sont confessionnelles, sont créées, la première en 1954 à Pira et la seconde en 1997 à Akpassi par l'Eglise catholique. Les deux écoles maternelles privées que compte la Commune sont Ayèkè de Koko, créée en 2000 par une expatriée suisse et Agongni à Lougba par l'ONG la Racine en 2010.

La Figure 6 montre la répartition spatiale de ces établissements scolaires. On s'aperçoit que la plupart des villages administratifs abritent au moins une école. Quelques-uns seulement des hameaux disposent d'une école. Toutes les écoles privées sont implantées dans les grosses agglomérations telles que Bantè, Gouka, Pira et Akpassi. L’engouement de création d'école avait gagné les fermes et hameaux qui ont aussi tenté, parfois sans succès. L'autorisation de création de nombreuses écoles 
primaires n'est obtenue que des années plus tard et a fini par être réglementée. C'est le cas des écoles de Tchambala, Fèro-Iya, Oké-Owo et Kadjogbé.

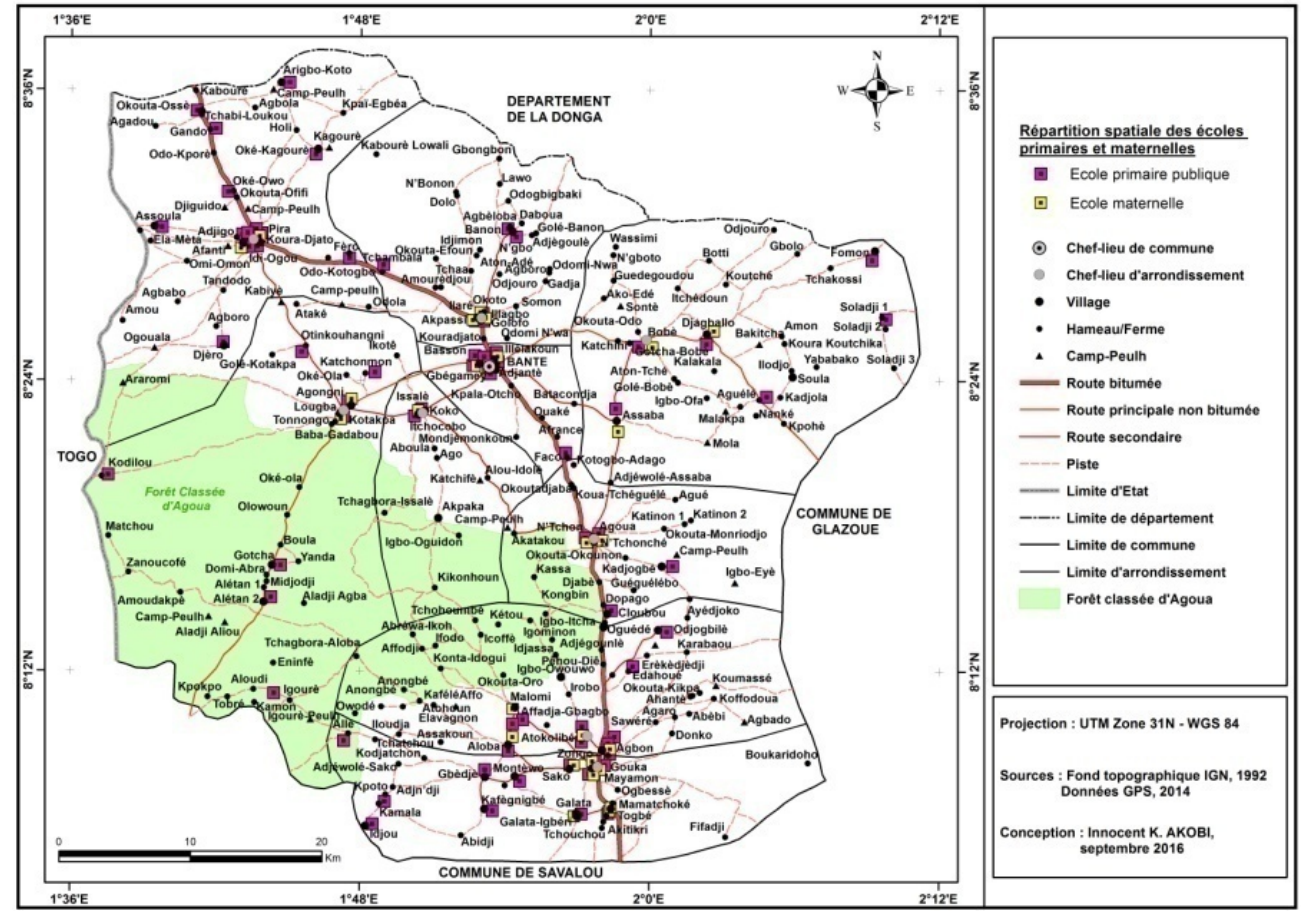

Figure 6. Répartition spatiale des écoles maternelles et primaires publiques et privées Source: Enquêtes de terrain, 2016

A lecture de cette carte, on s'aperçoit que la plupart des fermes et hameaux sont dépourvus d'école. Les quelques-uns qui en disposent sont confrontés à d'énormes difficultés.

$\checkmark \quad$ Le poids financiers des investissements publics dans l'éducation primaire à Bantè

La figure 7 montre l'importance des ressources investies au niveau de chaque arrondissement de la commune pour la construction et la réfection des modules de classe, la construction de latrines et la fabrication de mobiliers. L'inégalité des montants des investissements faits par arrondissement reflète surtout le poids démographique de chaque arrondissement et traduit l'importance des besoins d'investissement. Au total un milliard cinq cent cinquante-cinq millions quatre cent quatre-vingt-sept mille deux cent cinquante-six (1 555487256 ) francs CFA sont investis de 2008 à 2015 pour 124 salles de classe construites, 36salles de classe réfectionnées, 120 cabines de latrines construites et 3255 tables et bancs fabriqués par la mairie (Mairie de Bantè, 2016). 


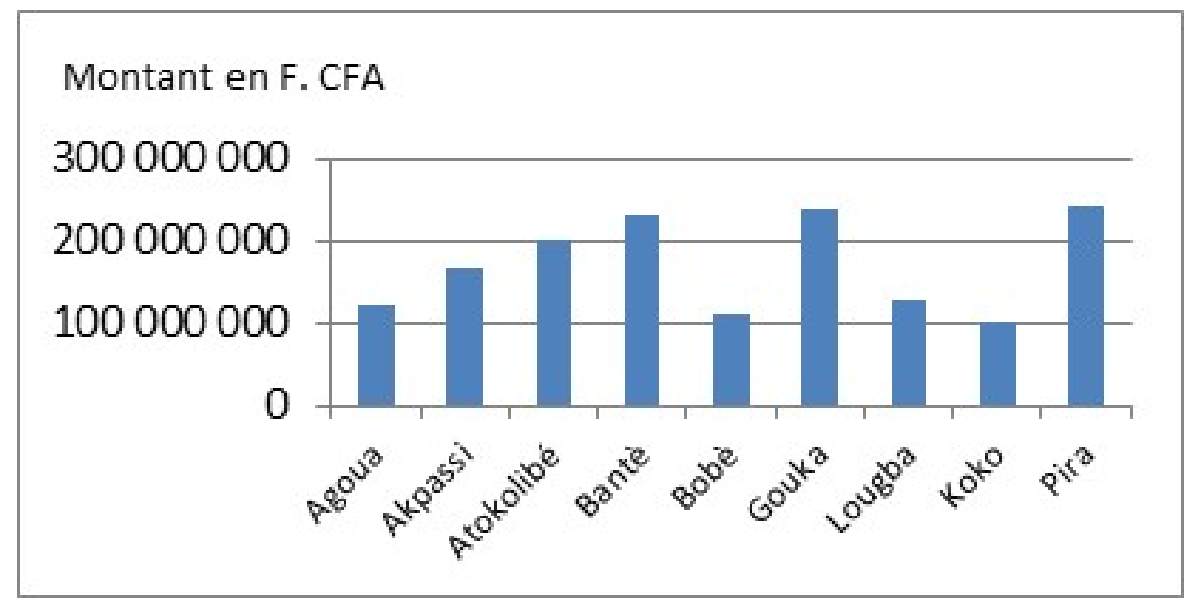

Figure 7. Montants investis par arrondissement pour les infrastructures scolaires Source: Mairie de Bantè, 2016

Les sources de ces financements faits par la mairie sont diverses et variées. On peut citer entre autres, les communautés, les ressources propres de la commune, les ressources transférées par l'Etat, les fonds de la coopération décentralisée (notamment entre le Département des Collines et le Conseil Régional de Picardie en France), les financements de divers Partenaires Techniques et Financiers notamment la Banque Mondiale, la Banque Islamique de Développement, l'Union Européenne, l'ONG RACINES, Japon 6, l’ONG PAEDRE, etc.

\section{Limites Spécifiques des Mesures Dans La Commune de Bantè : Les Contraintes Géographiques}

Elles peuvent s'apprécier sur plusieurs volets mais le plus important est celui de l'inaccessibilité géographique corollaire de la dynamique de peuplement spontané qui est spécifique à la Commune de Bantè que nous avons choisi d'analyser.

Il faut faire remarquer que toutes les écoles maternelles et primaires privées sont concentrées dans les grosses agglomérations telles que Bantè, Gouka, Pira et Akpassi. Par contre les écoles maternelles publiques sont réparties dans les principaux villages. Aucun hameau ne dispose d'école maternelle ; mais quelques-uns ont créé chacun une école primaire publique. De facto, il se pose un problème d'accessibilité aux infrastructures scolaires aux enfants des hameaux et fermes privés ou éloignés d'une école comme l'indique la figure 8. 


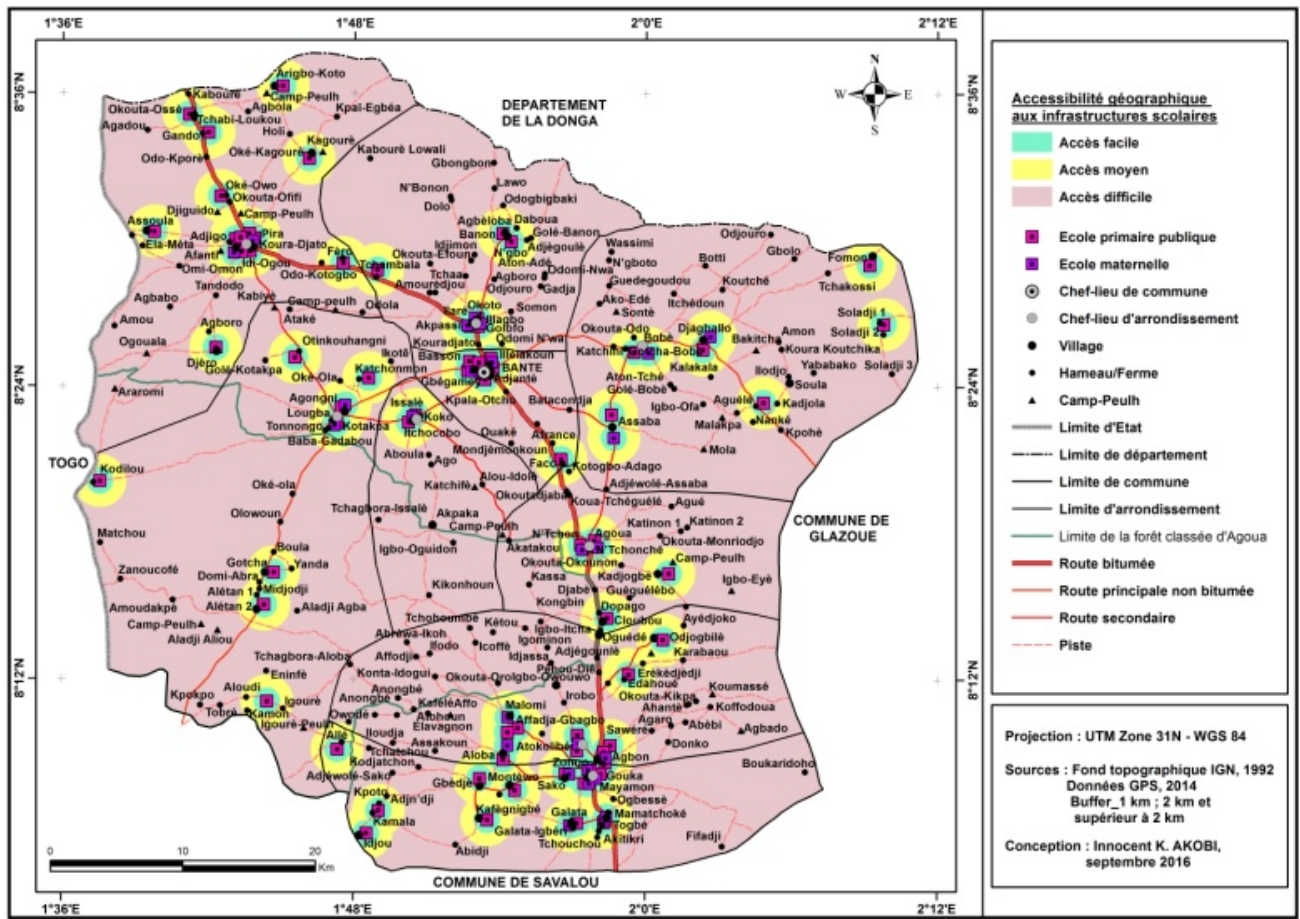

Figure 8. Niveaux d'accessibilité géographique aux infrastructures scolaires Source : Enquêtes de terrain, mai 2016

Selon les normes admises, est considérée comme accessible pour les élèves de la maternelle et du primaire, la distance de $1 \mathrm{~km}$; l'accessibilité moyenne est de $2 \mathrm{~km}$; au-delà, c'est l'accessibilité difficile. L'accessibilité difficile comme l'indique la figure 8 touche la plupart des fermes et hameaux dont les enfants n'ont pas la chance d'aller à l'école. Ce sont des localités totalement enclavées qui sont coupées des centres en temps de pluie, comme l'illustrent la Planche 1. 


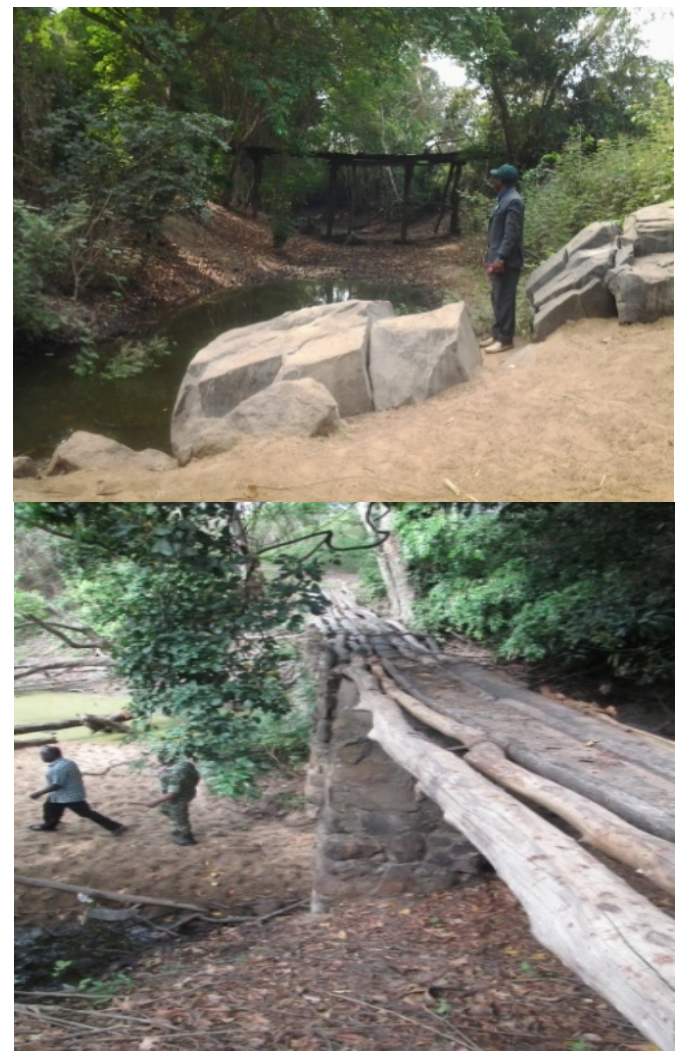

Planche 1.Le fleuve Zou sur l'axe Gbèdjè-Allé au loin, un pont de fortune en saison pluvieuse (à gauche) et le pont de fortune sur le fleuve Zou axe Gbèdjè-Idjou (à droite)

Prise de vue : Akobi, mars 2015
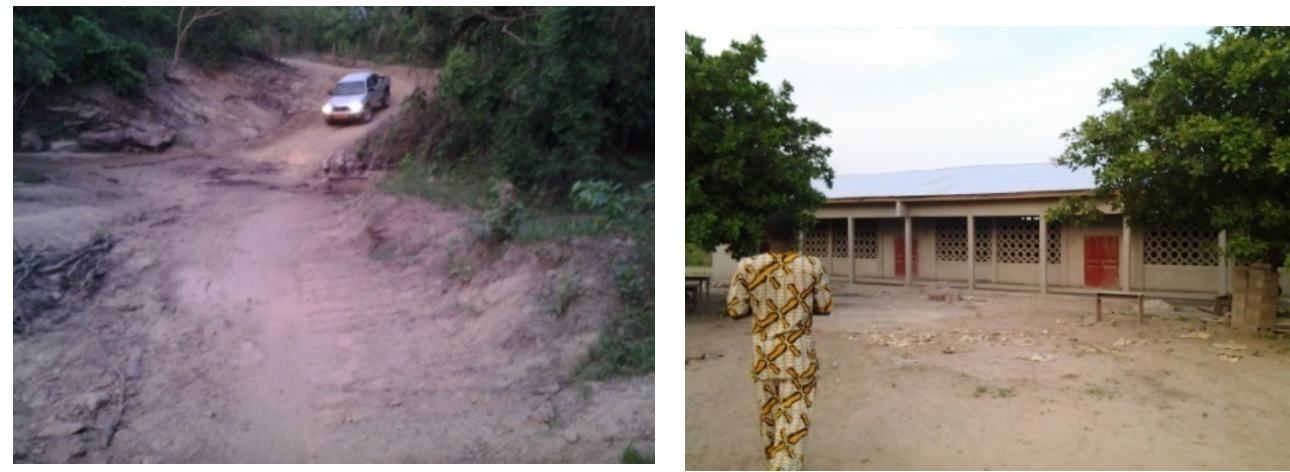

Planche 2. La traversée d'un affluent de Odjouro en direction de Fomon (à gauche) et la construction du module de 3 classes à Fomon, un chantier ayant souffert de l'enclavement Prise de vue : Akobi, janvier 2017

Les entreprises sélectionnées pour y réaliser les ouvrages scolaires éprouvent d'énormes peines faute d'ouverture de voie. Ce furent les cas des localités de Allé en 2010, de Kagourè, Adja-Pira et Kodilou en 2013, de Idjou et Fomon en 2016 (Photo 4) pour la construction des modules de classe 
de leur école primaire publique, ont témoigné les techniciens de la mairie et les autorités locales.

Dans cet ordre d'idées, il faut faire remarquer que l'école de Coulougbou dessert au moins 4 localités, tout comme Faco, Tchamballa, Aguélé, Fèro-Iya. Les écoles des hameaux tels que Allé, Idjou, Kamala, Oké-Owo, Fomon, Aguélé, Odjogbilè, Edahoué, Montèwo, Otikouangniéprouvent des difficultés d'effectif d'écoliers.Les enfants d'Igourè sont obligés de fréquenter l'école de Aloudi, une localitérevendiquée par la Commune de Savalou. Il y a de petites localités rapprochées qui pouvaient cogérer une école en mutualisant leurs ressources, mais qui abritent chacune une écoleà discontinuité éducative à cause du faible effectif des appranants. Le recrutement y est biennal, c'est-à-dire une fois tous les deux ans. Ces écoles manquent à la fois d'apprenants, d'enseignants, de locaux et de mobiliers (Photos 5, 6 et 7). C'est le cas des Ecoles Primaires Publiques des deux hameaux voisins Idjou et Kamala distants de $3 \mathrm{~km}$ (Planche 3 ).

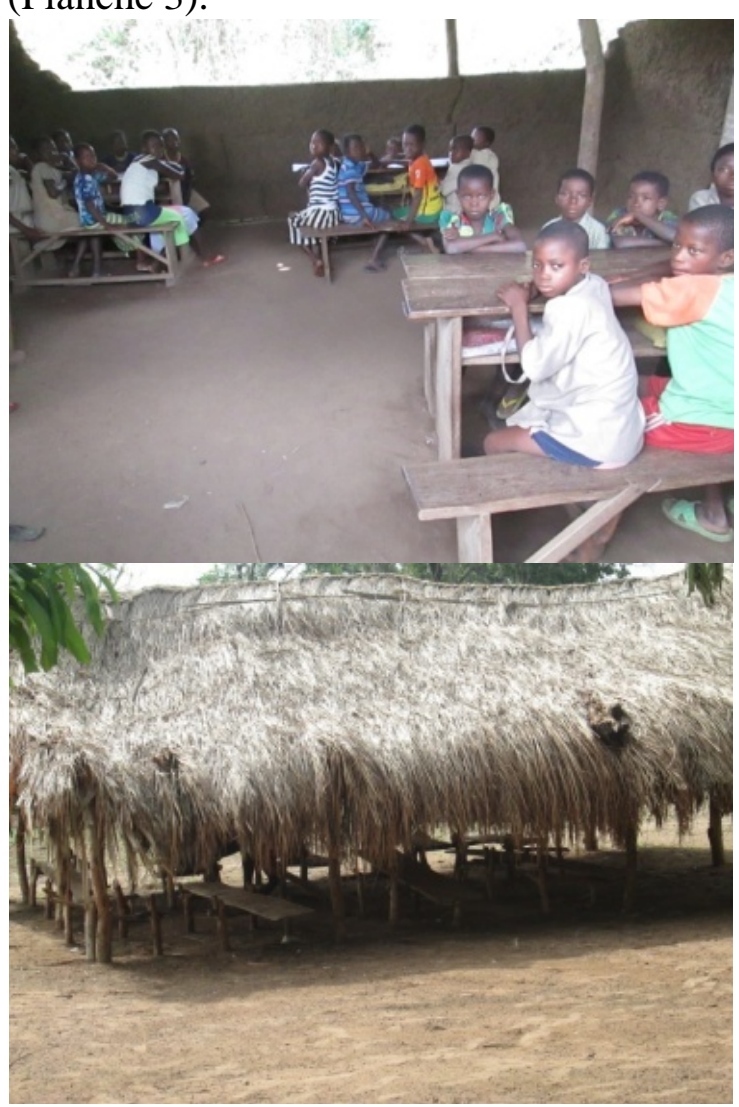

Planche 3. Les écoliers de l’EPP Kamala en classe (à gauche) et un local abritant l’EPP Idjou en matériaux précaires Prises de vues : Akobi, mars 2015 


\section{Impact des Contraintes Géographiques Sur Les Apprenants}

L'impact est essentiellement imatériel pour les apprenants; il est d'ordre physique, physiologique, moral et intellectuel.D'après les enquêtes de terrain, pour les 28000 écoliers iscrits au titre de l'année scolaire 20152016 aussi bien dans la maternelle qu'au primaire, dans le public que dans le privé, $73 \%$ ont une accessibilité facile, $13 \%$ une accessibilité moyenne et $14 \%$ une accessibilité difficile. Dans le lot des écoliers ayant une accessibilité moyenne et difficile, $80 \%$ résident dans les hameaux et les fermes qui n'abritent pas d'école. Les écoliers ayant l'accès difficle sont situés pour la plupart à une distance variant entre 2 et $4 \mathrm{~km}$. De facto, beaucoup d'entre eux ne rentrent pas à midi à la pause. Les plus endurants font la navette à pieds et parcourent en moyenne $10 \mathrm{~km}$ par jour à raison de $2,5 \mathrm{~km}$ pour un trajet. Cette situation influence négativement les résultats scolaires de ces apprenants sur plusieurs plans.

Le premier impact d'ordre phyqique et physiologique est lié à la fatigue de l'apprenant qui parcourt près de $10 \mathrm{~km}$ par jour. Le deuxième impact est relatif à la perte du temps. Il est obligé de quitter tôt la maison pour se rendre à l'école, et pour revenir tard. Il lui faut consacrer une demie heure en moyenne par trajet ; ce qui fait par jour deux heures de marche pour un enfant de 8 à 12 ans. Pendant lasaison pluvieuse, sa vulnérabilité s'aggrave ; il est exposé aux intempéries sur son trajet. Il court également des risques de mouiller, d'oublier, de perdre les fournitures scolaires dans ses différents mouvements, du retard et de sanction de la part de son enseignant et de ses parents. L'humiliation qui s'ensuit et le sentiment d'incapacité ressenti, constituent pour l'enfant des chocs psycologiques qui finissent par affecter son rendement. «Ce dernier finit souvent par anbandonner l'école... », a souligné un conseiller pédagogique.

Par ailleurs, certains enfants sont placés auprès de leurs grands parents ou d'une famille alliée pendant que leurs parents sont à la ferme durant des semaines. Ces enfants qui sont obligés de se séparer de leurs parents biologiques pour continuer leur scolarité se trouvent parfois en situation difficile et supportent mal cette rupture temporaire. Leur suivi en général et celui de leurs études se trouvent compromis; car ils sont abandonnés à eux-mêmes durant cette période. Il arrive que leurs besoins à satisfaire à l'immédiat attendent plus longtemps. Ne pouvant pas se confier à leur tuteur, ils sont obligés d'attendre le retour de leurs parents.

Par rapport à l'éloignement, il constitue un facteur non moins négligeable en matière de contrainte géographique. Certaines populations sont partagées entre les villages centraux et leurs localités périphériques situées à des distances très considérables. Cette situation d'ambivalence de résidence complique les bases réelles de programmation pour la réalisation d'équipements scolaires. Ces populations sont comptabilisées pour le compte 
des équipements scolaires de leurs villages administratifs mais les abandonnent pour se rendre à leur résidence secondaire qui en est dépourvue. De ce fait elles sont temporairement privées des services publics à la périphérie.

\section{Conclusion}

Le territoire de la Commune de Bantè est marqué par un peuplement dynamique. Aux peuples Isha et Ifè anciennement installés se sont ajoutés par vagues successives d'autres groupes socioculturels venus du sud, du centre, du nord Bénin et des pays voisins.

Ce peuplement à caractère rural basé sur des activités agropastorales est tributaire des facteurs physiques, humains et économiques relativement favorables. Il en a résulté une dissémination de nombreux lieux habités sur l'ensemble du territoire de cette Commune.

Si pendant longtemps ce peuplement a très peu préoccupé les autorités centrales, il n'en demeure pas vrai qu'à l'avènement de la décentralisation, la situation perdure avec les autorités locales. En effet, la gestion de proximité et les prérogatives conférées aux nouveaux élus locaux ont révélé les contraintes d'aménagement du territoire inhérentes à ce peuplement auxquelles ils doivent faire face pour combler les attentes des populations. La question de scolarisation en donne une illustration parfaite avec les difficultés d'accessibilité géographique des enfants à l'école sans oublier la pénurie d'enseignants plus marquée dans les milieux reculés.

\section{References:}

2. Adam K. S. \& Boko M. (1983). Le Bénin, Cotonou, Editions/Edicef, Sodimas, $95 \mathrm{p}$.

3. Akobi K. I. (1997). Les migrations de population et leur impact socio-économique dans la Sous-préfecture de Bantè, mémoire de maîtrise de géographie, Université Nationale du Bénin, 135 p.

4. Akobi K. I. (2002). Question foncière et gestion des ressources naturelles au Moyen-Bénin, mémoire de DEA de géographie, Université Lumière Lyon 2, France, 92 p.

5. Arouna O. (2012). Cartographie et modélisation prédictive des changements spatio-temporels de la végétation dans la Commune de Djidja au Bénin : implications pour l'aménagement du territoire. Thèse de Doctorat, Université d’Abomey-Calavi, Bénin, 246 p.

6. Baudelle G. (2000). Géographie du peuplement, Paris, Armand Colin, $192 p$.

7. Cornévin R. (1965). Histoire du Dahomey, Edition Berger Levraut, $568 \mathrm{p}$. 
8. Droy I. (2005). Pauvreté, enclavement et accès à l'école primaire en milieu rural guinéen. Mondes en développement, $\mathrm{N}^{\circ} 132$, pp. 111127.

9. Gomez C.A, Agoïnon N., Akobi K. I., \& Houssou S.C. (2016). Déterminants de l'accès à l'eau potable dans la Commune de Bantè, in Revue de Géographie Tropicale et d’Environnement, n², pp 106118.

10. INSAE (2016). Recensement Général de la Population et de l'Habitation (RGPH4). Résultats définitifs. Cotonou, Bénin, 209 p.

11. Mairie de Bantè (2016). Bilan des actions du septennat 2008-2015 du conseil communal de la deuxième mandature, 32 p.

12. Moupou M. (2010). Fronts pionniers dans le Cameroun méridional : de nouveaux territoires en mutation rapide. In IGUE O. J. et al, Maîtrise de l'espace et développement en Afrique. Etat des lieux. Editions Karthala. 340 p.

13. NotokpèK. S. (2010). Occupation du sol et dynamique de l'espace rural dans le bassin du haut Mono (Togo)'”, in Atta K. et Zoungrana P. T., Logiques paysannes et espaces agraires en Afrique. Editions Karthala. 380 p.

14. Oloukoï J. (2012). Utilité de la télédétection et des systèmes d'information géographique dans l'étude de la dynamique spatiale de l'occupation des terres au centre du Bénin. Thèse de Doctorat, Université d'Abomey-Calavi, Bénin, 304p.

15. ONU Bénin (2016). Evaluation des progrès accomplis par le Bénin dans la réalisation des OMD 2000-2015, PNUD Bénin, 174 p.

16. Pinchemel P. G. (1997). La face de la terre. Eléments de géographie, $5^{e}$ édition, Paris, Armand Colin/Masson, 517 p.

17. SCAD Bantè (2014). Rapport annuel des productions agricoles de la Commune de Bantè.

18. Sounon Bouko B. (2011). Colonisation agricole et dégradation $d u$ couvert végétal dans le secteur Wari-Maro-Igbomakro au Bénin. Thèse de Doctorat, Université d'Abomey-Calavi,

19. Thomas O. (2014). Cartographie et stratégie territoriale: retour d'expériences et modèle pour le développement durable du département des Collines. Thèse de Doctorat, Université d'AbomeyCalavi, Bénin, 288 p.

20. Touré T. A. (2010). Problématique maîtrise de l'espace et du développement en Afrique : quel rôle pour la télédétection spatiale et les systèmes d'information géographique? Systèmes spatiaux et structures régionales en Afrique, (édition Karthala). 